\title{
Peculiarities of the magnetocaloric properties in Ni-Mn-Sn ferromagnetic shape memory alloys
}

\author{
V. V. Khovaylo, ${ }^{1,2}$ K. P. Skokov, ${ }^{3,4}$ O. Gutfleisch, ${ }^{4}$ H. Miki, ${ }^{5}$ T. Takagi, ${ }^{5}$ T. Kanomata, ${ }^{6}$ V. V. Koledov, ${ }^{7}$ \\ V. G. Shavrov, ${ }^{7}$ G. Wang, ${ }^{8}$ E. Palacios, ${ }^{8}$ J. Bartolomé, ${ }^{8}$ and R. Burriel ${ }^{8}$ \\ ${ }^{1}$ National University of Science and Technology "MISiS," Moscow 119049, Russia \\ ${ }^{2}$ Physics Department, Moscow State Mining University, Moscow 119991, Russia \\ ${ }^{3}$ Faculty of Physics, Tver State University, Tver 170000, Russia \\ ${ }^{4}$ Institute for Metallic Materials, Leibniz Institute for Solid State and Materials Research Dresden (IFW Dresden), P.O. Box 270016, \\ D-01171 Dresden, Germany \\ ${ }^{5}$ Institute of Fluid Science, Tohoku University, Sendai 980-8577, Japan \\ ${ }^{6}$ Faculty of Engineering, Tohoku Gakuin University, Tagajo 985-8537, Japan \\ ${ }^{7}$ V.A. Kotel'nikov Institute of Radioengineering and Electronics, Moscow 125009, Russia \\ ${ }^{8}$ Instituto de Ciencia de Materiales de Aragón and Departamento de Fisica de la Materia Condensada, CSIC-Universidad de Zaragoza, \\ Zaragoza 50009, Spain
}

(Received 17 November 2009; revised manuscript received 18 March 2010; published 7 June 2010)

\begin{abstract}
Magnetocaloric properties of a $\mathrm{Ni}_{50} \mathrm{Mn}_{36} \mathrm{Co}_{1} \mathrm{Sn}_{13}$ ferromagnetic shape memory alloy have been studied experimentally in the vicinity of a first-order magnetostructural phase-transition low-temperature paramagnetic martensite $\leftrightarrow$ high-temperature ferromagnetic austenite. The magnetic entropy change $\Delta S_{m}$ calculated from the magnetization $M(T)$ data measured upon cooling is higher than that estimated from $M(T)$ measured upon heating. Contrary to $\Delta S_{m}$, the adiabatic temperature change $\Delta T_{a d}$ measured upon cooling is significantly smaller than that measured upon heating. The apparent discrepancy between $\Delta S_{m}$ and $\Delta T_{a d}$ (larger $\Delta S_{m}$, smaller $\Delta T_{a d}$ upon cooling, and smaller $\Delta S_{m}$, larger $\Delta T_{a d}$ upon heating) is caused by the hysteretical behavior of this magnetostructural transition, a feature common for all the alloys in the family of $\mathrm{Ni}_{50} \mathrm{Mn}_{25+x} Z_{25-x}$ $(Z=\mathrm{In}, \mathrm{Sn}, \mathrm{Sb})$ ferromagnetic shape memory Heusler compounds. The hysteresis causes the magnetocaloric parameters to depend strongly on the temperature and field history of the experimental processes.
\end{abstract}

DOI: 10.1103/PhysRevB.81.214406

PACS number(s): 75.30.Sg, 75.50.Cc

\section{INTRODUCTION}

Recently, a considerable interest has been paid to Mn-rich $\mathrm{Ni}_{50} \mathrm{Mn}_{25+x} Z_{25-x}(Z=\mathrm{Sn}, \mathrm{In}, \mathrm{Sb})$ Heusler alloys that undergo a thermoelastic martensitic transformation from a hightemperature cubic austenitic phase to a low-temperature monoclinic or orthorhombic martensitic phase. ${ }^{1}$ A specific feature of these alloys is that the saturation magnetization is greatly reduced or becomes almost zero upon the structural transformation from austenite to martensite. ${ }^{2-11}$ The origin of this was suggested to be the strengthening of antiferromagnetic interactions caused by an abrupt change in the Mn-Mn interatomic distances occurring upon the martensitic transformation.

The strong interrelation between crystal structure and magnetism is responsible for a large inverse magnetocaloric effect (MCE) in $\mathrm{Ni}_{50} \mathrm{Mn}_{25+x} Z_{25-x}$ (Refs. 12-14). Most of the papers devoted to the MCE of these alloys report on the isothermal entropy change $\Delta S_{m}$ calculated from magnetization measurements using the Maxwell relation $(\partial S / \partial H)_{T}$ $=(\partial M / \partial T)_{H}$. Hereafter, $\Delta S_{m}$ is defined as the entropy change upon variation in the applied field at a constant temperature, and it includes the magnetic, electronic, and structural contributions; the two latter ones may actually be larger than that stemming from the magnetic subsystem. ${ }^{15}$ For the $Z=$ Sn system, values of $\Delta S_{m}$ up to $30 \mathrm{~J} / \mathrm{kg} \mathrm{K}$, for the magnetic field change $\Delta H=5 \mathrm{~T}$, have been reported in the vicinity of the first-order martensitic transformation. ${ }^{12,16-18}$ Moya et al. ${ }^{19}$ performed differential scanning calorimetry measurements upon warming, determining $\Delta S_{m}$ in a $\mathrm{Ni}_{50} \mathrm{Mn}_{35} \mathrm{Sn}_{15}$ compo- sition under magnetic fields up to $3 \mathrm{~T}$. They found that $\Delta S_{m}$ obtained from these measurements is in a good agreement with the value calculated from the Maxwell relation. ${ }^{12}$

It is worth noting that the MCE in the vicinity of the martensitic transition of $\mathrm{Ni}_{50} \mathrm{Mn}_{25+x} Z_{25-x}$ has been studied so far mostly for heating runs, i.e., for the case of the reverse transformation from the martensitic to the austenitic state. However, characteristic temperatures of the direct martensitic transformation are lower than those of the reverse one. In fact, this affects the magnitude of the magnetization change across the transitions. Moreover, the direct martensitic transformation is an exothermic process whereas the reverse one is endothermic. Evidently, the magnetocaloric properties of a ferromagnetic shape memory alloy upon direct and reverse transformations will not be the same. What is more important, a large hysteresis of the martensitic transitions confers an irreversible character to the physical variables across the transition. It makes the physical measurements to depend strongly on the temperature and field history of the processes in the transition region. This dependence profoundly affects the deduced values for the magnetocaloric parameters. This essential effect has not been properly considered in previous reports. In order to shed light on these issues we undertook a comparative study of the isothermal magnetic entropy change $\Delta S_{m}$ and the adiabatic temperature change $\Delta T_{a d}$ during heating and cooling runs in a Co-doped $\mathrm{Ni}_{50} \mathrm{Mn}_{37-x} \mathrm{Co}_{x} \mathrm{Sn}_{13}$ alloy.

The transition temperature from austenite to martensite is frequently denoted as $T_{A M}$ and the reverse transition as $T_{M A}$. Generally, martensitic transformations are characterized by martensite start and finish, $M_{s}$ and $M_{f}$, and austenite start and 
finish, $A_{s}$ and $A_{f}$, temperatures. They can be attributed to the onset and the end of the rapid change in magnetization seen on cooling and heating curves, respectively. Evidently, $M_{f}<T_{A M}<M_{s}$ and $A_{s}<T_{M A}<A_{f}$. The Curie temperatures of the austenite and martensite are denoted by $T_{C}^{A}$ and $T_{C}^{M}$, respectively, with $T_{C}^{M}<T_{C}^{A}$.

The choice of the selected composition, $\mathrm{Ni}_{50} \mathrm{Mn}_{37-x} \mathrm{Co}_{x} \mathrm{Sn}_{13}$, is based on the following arguments. In the $\mathrm{Ni}_{50} \mathrm{Mn}_{25+x} \mathrm{Sn}_{25-x}$ compounds the proportion of $\mathrm{Sn}$ strongly varies $T_{M A}$ and $T_{A M}$, which are very dependent on the valence electron concentration. ${ }^{20}$ Since, for application purposes, it is desirable to have the transition from a weakly magnetic martensite to a ferromagnetic austenite near room temperature, we have selected the composition $x=12$ as best suited. ${ }^{20}$ Besides, a high magnetization jump at the martensitic transition is advisable to get a large and inverse MCE, which can be achieved if $T_{C}^{M}$ is lower than the martensitic transition start $A_{s}$, and $T_{C}^{A}$ is much higher than the martensitic transition finish $A_{f}$, that is, if $T_{C}^{M}<A_{s}<A_{f} \ll T_{C}^{A}$. The Curie temperature of the austenite $T_{C}^{A}$ depends very weakly on the $\mathrm{Sn}-\mathrm{Mn}$ proportion, but can be increased by doping with a small amount of $\mathrm{Co}$, as reported for the In and $\mathrm{Sb}$ Heusler alloys. ${ }^{8,21}$ Moreover, this Co doping also increases the magnetization of the austenite, favoring a high MCE at the structural transition. ${ }^{21}$ Thus, approaching $T_{A M}$ and $T_{M A}$ to room temperature, on one side, and increasing the magnetization of the austenite phase, constitutes the criteria of choice of the sample composition.

\section{SAMPLE PREPARATION AND MEASUREMENTS}

A polycrystalline ingot of the $\mathrm{Ni}_{50} \mathrm{Mn}_{36} \mathrm{Co}_{1} \mathrm{Sn}_{13}$ nominal composition was prepared by conventional arc-melting. Since the weight loss after the melting was negligible, the real composition was assumed to be almost identical to the nominal one. The ingot was annealed in vacuum at $1173 \mathrm{~K}$ for $24 \mathrm{~h}$ and quenched in water. Samples for magnetization and adiabatic temperature change measurements were cut from the core part of the ingot. Magnetization measurements were performed in magnetic fields up to $2 \mathrm{~T}$ in a Quantum Design superconducting quantum interference device magnetometer. Direct measurements of the adiabatic temperature change $\Delta T_{a d}$ were performed in an experimental setup described in Ref. 22. The magnetic field was produced by a permanent magnet on the basis of a Halbach cylindrical structure. The maximum field in the bore center was $\mu_{0} H$ $=1.93 \mathrm{~T}$. The magnetic field change rate was $2 \mathrm{~T} / \mathrm{s}$ that allows one to prevent heat losses from the specimen during the measurements. The temperature of the sample was monitored with accuracy better than $0.02 \mathrm{~K}$ by a Copper-Constantan thermocouple which was in direct contact with the sample. During the measurements, the target temperature was approached without overheating/overcooling.

\section{EXPERIMENTAL RESULTS AND DISCUSSION}

Shown in Fig. 1 is the temperature dependence of the magnetization, $M(T)$, measured at a magnetic field $\mu_{0} H$ $=0.1 \mathrm{~T}$ upon heating and cooling. Upon heating, $M(T)$ ini-

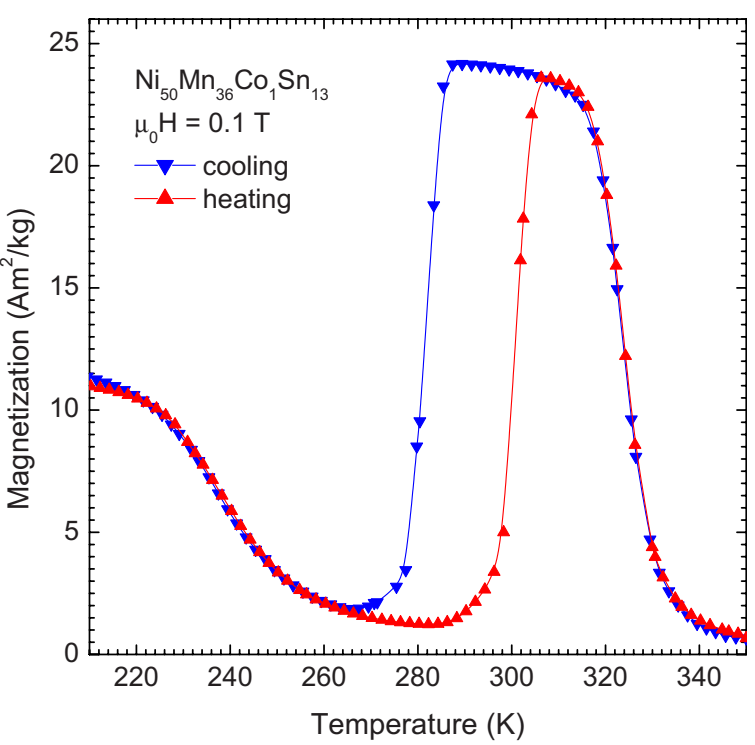

FIG. 1. (Color online) Magnetization curve of $\mathrm{Ni}_{50} \mathrm{Mn}_{36} \mathrm{Co}_{1} \mathrm{Sn}_{13}$ measured during cooling and heating in a magnetic field $\mu_{0} H$ $=0.1 \mathrm{~T}$.

tially decreases and then increases drastically due to the structural phase transition from martensitic to austenitic phase. Recently, it has been verified experimentally ${ }^{23,24}$ that the decrease in magnetization observed in the lowtemperature martensitic phase of $\mathrm{Ni}_{50} \mathrm{Mn}_{25+x} Z_{25-x}$ is caused by the transition from magnetically ordered to paramagnetic state, i.e., corresponds to the Curie temperature of the martensitic phase $T_{C}^{M}$. The rapid decrease in the magnetization seen at still higher temperature corresponds to a magnetic transition of the austenitic phase from ferromagnetic to paramagnetic state, i.e., corresponds to the Curie temperature of the austenitic phase $T_{C}^{A}$. The Curie temperatures of the martensitic and austenitic phases, determined as the minima on $(\partial M / \partial T)_{H}$, were found to be $T_{C}^{M}=238 \mathrm{~K}$ and $T_{C}^{A}=325 \mathrm{~K}$, respectively. In the vicinity of $T_{C}^{M}$ and $T_{C}^{A}$, the cooling and heating curves coincide, indicating that the magnetic phase transitions are of second order. A temperature hysteresis of about $16.5 \mathrm{~K}$ on the structural transition between the heating and cooling curves is due to the first-order martensitic phase transition. Characteristic temperatures of the direct and reverse martensitic transformations determined in the same manner as $T_{C}^{M}$ and $T_{C}^{A}$ were found to be $T_{A M}=284 \mathrm{~K}$ and $T_{M A}=300.5 \mathrm{~K}$, respectively.

The temperature dependence of the magnetization, $M(T)$, measured upon heating and cooling at various magnetic fields in the vicinity of the martensitic phase transition is shown in Fig. 2. The external magnetic field shifts the martensitic transition toward lower temperatures, thus stabilizing the ferromagnetic austenitic phase. As the magnetic field changes from $\mu_{0} H=0.1$ to $2 \mathrm{~T}$, the direct martensitic transformation temperature shifts by $\delta T_{A M} \approx-3.8 \mathrm{~K}$, whereas the reverse martensitic transformation temperature shifts by $\delta T_{M A} \approx-2.9 \mathrm{~K}$. The shift of $T_{A M}$ is slightly larger due to the fact that, for the fields $\mu_{0} H>0.1 \mathrm{~T}$, the magnetization change, $\Delta M$, (hence, the Zeeman energy $\Delta M \cdot H$ ) during the direct martensitic transformation is larger than the corre- 


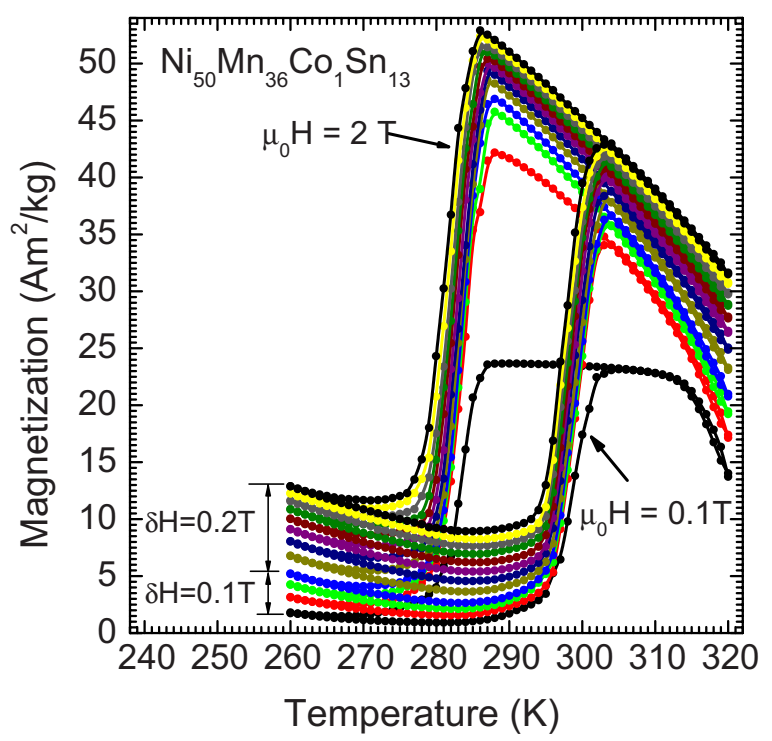

FIG. 2. (Color online) Temperature dependence of the magnetization in the vicinity of the martensitic phase transition of $\mathrm{Ni}_{50} \mathrm{Mn}_{36} \mathrm{Co}_{1} \mathrm{Sn}_{13}$ at various magnetic fields.

sponding change during the reverse transformation.

The isothermal entropy change $\Delta S_{m}$ calculated from the $M(T)$ dependencies using the Maxwell relation $(\partial S / \partial H)_{T}$ $=(\partial M / \partial T)_{H}$ is depicted in Fig. 3. The plot of $\Delta S_{m}$, calculated from the $M(T)$ curves measured upon heating, exhibits a peak at the reverse martensitic transformation temperature $T_{M A}$. Correspondingly, $\Delta S_{m}$ calculated from the $M(T)$ curves measured upon cooling exhibits a peak at the direct martensitic transformation temperature $T_{A M}$. For a magnetic field change from 0 to $\mu_{0} H=2 \mathrm{~T}$, the peak value of $\Delta S_{m}$ at the direct martensitic transformation temperature, $\Delta S_{m}^{\text {cooling }}$ $=13.8 \mathrm{~J} / \mathrm{kg} \mathrm{K}$, is larger than the value at the reverse transformation, $\Delta S_{m}^{\text {heating }}=10.4 \mathrm{~J} / \mathrm{kg} \mathrm{K}$. This difference was to be expected because the magnetization change, $\Delta M$, is larger

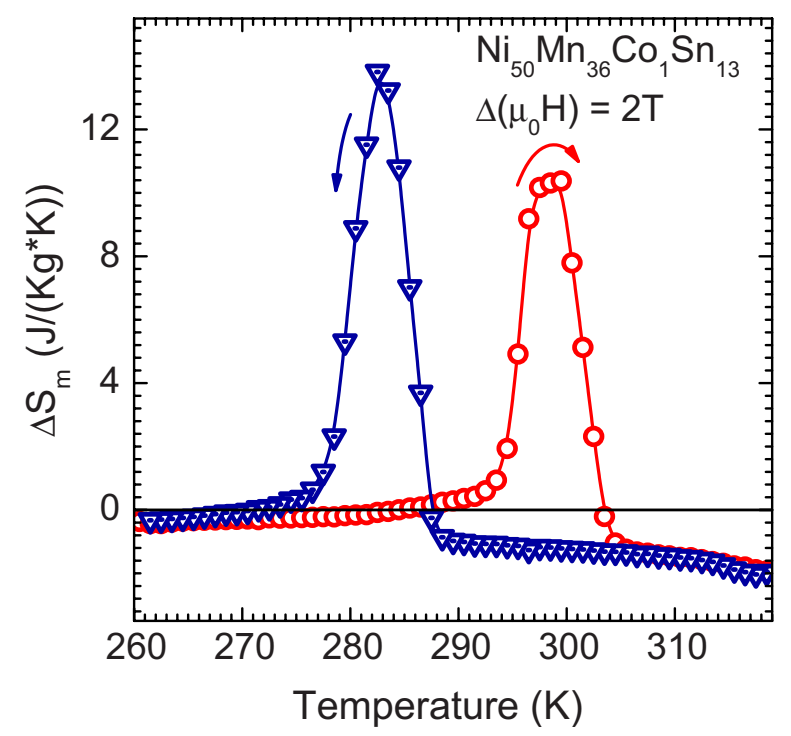

FIG. 3. (Color online) Isothermal magnetic entropy change, $\Delta S_{m}$, in $\mathrm{Ni}_{50} \mathrm{Mn}_{36} \mathrm{Co}_{1} \mathrm{Sn}_{13}$, calculated from $M(T)$ measured upon cooling (triangles) and heating (circles).

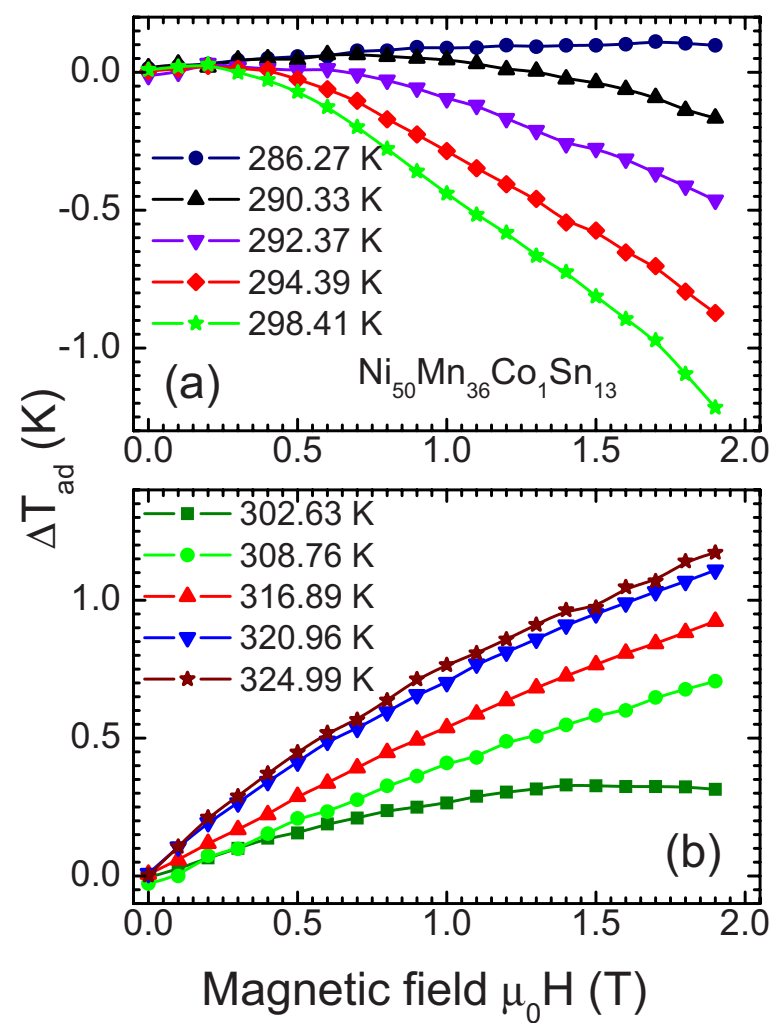

FIG. 4. (Color online) Magnetic field dependence of the adiabatic temperature change in $\mathrm{Ni}_{50} \mathrm{Mn}_{36} \mathrm{Co}_{1} \mathrm{Sn}_{13}$ measured upon heating protocol in the vicinity (a) of the magnetostructural transition and (b) of the Curie temperature of the austenitic phase.

upon the direct martensitic transformation and both transitions have a similar temperature width. The noticeably higher value of $\Delta M$ at the direct martensitic transformation with respect to the reverse one is due to the closeness of these transitions to $T_{C}^{A}$ since the magnetization of the austenite phase depends strongly on temperature in this range close to the Curie temperature. The curve of $\Delta S_{m}$ calculated from the isothermal $M(H)$ measurements was found to be practically the same.

Shown in Fig. 4 are examples of the field dependence of the adiabatic temperature change measured at fixed temperatures in the vicinity of the first-order structural (martensitic) phase transition [Fig. 4(a)] and of the second-order magnetic phase transition of the austenite, $T_{C}^{A}[$ Fig. 4(b)]. The measurements were performed at increasing temperatures; the magnetic field changes from 0 to $\mu_{0} H=1.9 \mathrm{~T}$. These results clearly demonstrate that $\mathrm{Ni}_{50} \mathrm{Mn}_{36} \mathrm{Co}_{1} \mathrm{Sn}_{13}$ exhibits an inverse MCE at the martensitic phase transition [Fig. 4(a)] and a conventional MCE at the austenite Curie temperature $T_{C}^{A}$ [Fig. 4(b)].

The temperature dependence of the adiabatic temperature change measured for the magnetic field change from 0 to $\mu_{0} H=1.9 \mathrm{~T}$ at increasing temperatures, $\Delta T_{a d}^{\text {heating }}$, and at decreasing temperatures, $\Delta T_{a d}^{\text {cooling }}$, is shown in Fig. 5. At temperatures below $\sim 275 \mathrm{~K}$ and above $308 \mathrm{~K}$, i.e., beyond the martensitic phase-transition region, the $\Delta T_{a d}^{\text {heating }}(T)$ and $\Delta T_{a d}^{\text {cooling }}(T)$ curves coincide. A peak with a maximum $\Delta T_{a d}$ $\approx 1.15$ at $325 \mathrm{~K}$, seen on both heating and cooling curves, 


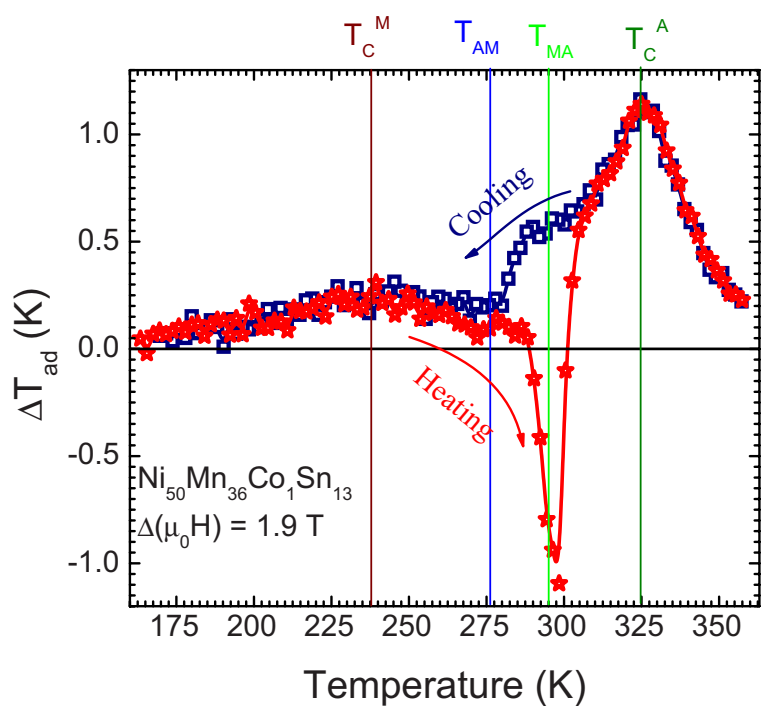

FIG. 5. (Color online) Temperature dependence of the adiabatic temperature change, $\Delta T_{a d}$, in $\mathrm{Ni}_{50} \mathrm{Mn}_{36} \mathrm{Co}_{1} \mathrm{Sn}_{13}$ upon cooling (triangles) and heating (circles). Vertical lines denote Curie temperature of the martensitic and austenitic phases, $T_{C}^{M}$ and $T_{C}^{A}$, respectively, and characteristic temperatures of the direct and reverse martensitic transformations, $T_{A M}$ and $T_{M A}$. Values of $T_{C}^{M}$ and $T_{C}^{A}$ are taken from Fig. 1. Values of $T_{A M}$ and $T_{M A}$ are extracted from Fig. 2 for the magnetic field $\mu_{0} H=2 \mathrm{~T}$.

corresponds to the Curie temperature of the austenitic phase $T_{C}^{A}$. A broad and weak anomaly with a maximum $\Delta T_{a d}$ $\approx 0.32 \mathrm{~K}$ at $T \approx 240 \mathrm{~K}$ is associated with the Curie temperature of the martensitic phase $T_{C}^{M}$. Within the temperature interval of the martensitic phase transition, the adiabatic temperature change exhibits hysteresis. On heating, the reverse martensitic transformation is accompanied by a well-defined negative peak of the adiabatic temperature change in the vicinity of $T_{M A}$. During the heating process, $\Delta T_{a d}^{\text {heating }}(T)$ reaches the value $-1.1 \mathrm{~K}$, which is significantly higher in the studied alloy than the value reported for a $\mathrm{Ni}_{48.3} \mathrm{Mn}_{37.5} \mathrm{Sn}_{14.2}$ composition $\left(\Delta T_{a d} \approx-0.3 \mathrm{~K}\right.$ for a magnetic field change from 0 to $\left.\mu_{0} H=3 \mathrm{~T}\right){ }^{25}$

The behavior of $\Delta T_{a d}$ measured in the temperature interval of the martensitic transformation upon heating and cooling is of particular interest. In principle, one would expect $\Delta T_{a d}$ to be proportional to $\Delta S_{m}{ }^{26}$ Then, the temperature dependence of the adiabatic temperature change would mimic the dependence of the isothermal magnetic entropy change and, therefore, a pronounced peak of $\Delta T_{a d}$ would be observed for both heating and cooling processes. Contrary to the expectation, $\Delta T_{a d}$ measured upon cooling exhibits only a weak anomaly at $T_{A M}$ (Fig. 5) despite the fact that $\Delta S_{m}^{\text {cooling }}$ is larger than $\Delta S_{m}^{\text {heating }}$. Moreover, $\Delta T_{a d}$ measured upon cooling, while showing a dip in the temperature interval of the direct martensitic transformation (Fig. 5), does not reach a negative value, which would be reasonable to expect in the case of inverse magnetocaloric effect.

The results can be explained considering the paths of the experimental processes in the phase diagram of the compound representing $\mu_{0} H$ vs $T$. Figure 6 depicts the phase diagram of the martensite $\leftrightarrow$ austenite transition, as deduced

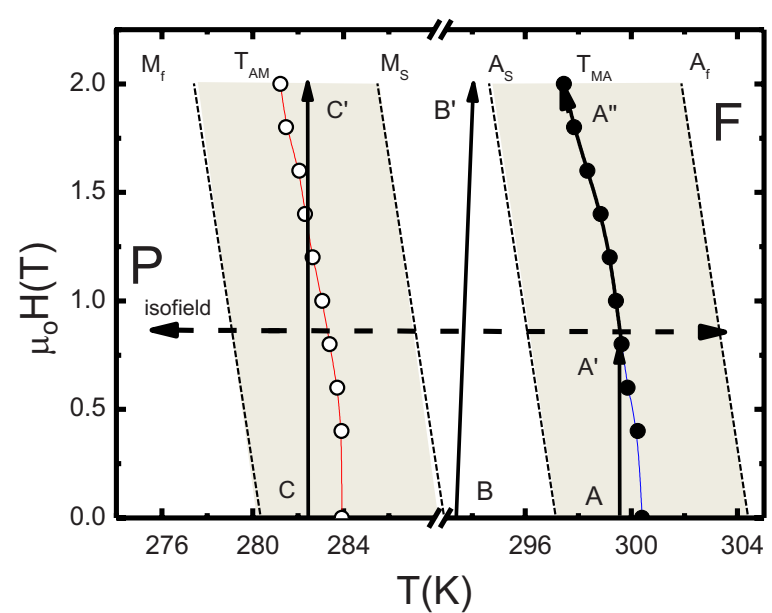

FIG. 6. (Color online) Magnetic phase diagram of $\mathrm{Ni}_{50} \mathrm{Mn}_{36} \mathrm{Co}_{1} \mathrm{Sn}_{13}$ determined from $M(T)$ measurements upon heating (full circles) and cooling (empty circles). P denotes paramagnetic martensite, F denotes ferromagnetic austenite. The thick lines show the different processes followed by the system in adiabatic measurements of $\Delta T_{a d}$ (see text). The dashed arrowed line corresponds to one of the isofield measurements represented in Fig. 2. The shadowed areas show the finite width of the phase-transitions bands.

from the points of maximum slope of the magnetization curves shown in Fig. 2. For processes with increasing temperature or increasing field, the phase boundary between the paramagnetic and the ferromagnetic phases is the $T_{M A}$ line. On the contrary, for decreasing temperature or field, the corresponding phase boundary is the $T_{A M}$ line. To be precise, there is a broad transition band in the field-temperature diagram around each of these lines.

For the determination of $\Delta S_{m}$, represented in Fig. 3, the paths followed in the isofield $M(T)$ measurements have been represented in the phase diagram of Fig. 6 with the dashed horizontal line. On heating, there is a maximum of $\Delta S_{m}$ when crossing the $T_{M A}$ transition line and, when cooling, the maximum happens at the crossing of the $T_{A M}$ transition line.

The $\Delta T_{a d}$ results represented in Figs. 4 and 5 can be explained with the following arguments. When $\Delta T_{a d}$ is measured during a heating protocol in the martensitic phase, the values of $\Delta T_{a d}^{\text {heating }}$, represented in Fig. 5, have a small maximum around the Curie temperature of the martensite $T_{C}^{M}$ $=238 \mathrm{~K}$. Then, above $238 \mathrm{~K}, \Delta T_{a d}^{\text {heating }}$ decreases to a small value, due to the very slight effect of the magnetic field in the paramagnetic state. The application of a magnetic field at a temperature in the $A_{s}<T<A_{f}$ interval easily converts the paramagnetic martensite into ferromagnetic austenite. At a point such as A of Fig. 6 , close to the $T_{M A}$ transition line, the adiabatic process for increasing field can be separated in two steps, (i) in the $\mathrm{A}-\mathrm{A}^{\prime}$ segment, the sample is mostly paramagnetic and there is practically no change in $\Delta T_{a d}^{\text {heating }}$, (ii) the phase transition from martensite to austenite takes place at constant entropy following the $T_{M A}$ transition line from $\mathrm{A}^{\prime}$ to $\mathrm{A}^{\prime \prime}$ and the system decreases its temperature. As a consequence, $\Delta T_{a d}^{\text {heating }}$ exhibits a sharp negative peak in the vicinity of the reverse martensitic transition, as is experimentally observed. For a more realistic description of our case, the 
width of the transition region has to be considered, producing more gradual variation in the adiabatic trajectory $\mathrm{A}$ $-\mathrm{A}^{\prime}-\mathrm{A}^{\prime \prime}$. At temperatures above $A_{f}$, the ferromagnetic austenite shows positive $\Delta T_{a d}^{\text {heating }}$ values, peaking at $T_{C}^{A}$ $=325 \mathrm{~K}$ and, then, decreasing gradually, as happens in every typical ferromagnetic to paramagnetic transition.

When the system is cooled, coming from the ferromagnetic austenite, the phase will be stable down to $M_{s}$ and, therefore, the contribution from the structural subsystem to $\Delta T_{a d}$ will not be observed above this temperature. At point $\mathrm{B}$, coming from high temperature, the sample remains in the austenite phase, so in the process $\mathrm{B}-\mathrm{B}^{\prime}$ there is only a weak positive $\Delta T_{a d}^{\text {cooling }}$ due to the field dependence of the ferromagnetic state. If the system is cooled to the temperature interval of the direct transformation, $M_{f}<T<M_{s}$, the sample transits to the martensite phase. In a subsequent adiabatic process, the trajectory of the system, with increasing magnetic field, follows the line $\mathrm{C}-\mathrm{C}^{\prime}$. Since the formed martensitic phase is thermodynamically stable up to the $A_{s}$ transition line, a rather strong magnetic field would be required to initiate the transformation from paramagnetic martensite to ferromagnetic austenite. Taking into account the sensitivity of the reverse martensitic transformation temperature $T_{M A}$ to the applied magnetic field, $d T_{M A} / d H \approx-1.5 \mathrm{~K} / \mathrm{T}$, and the temperature hysteresis of the martensitic transformation, $\sim 16.5 \mathrm{~K}$, the critical magnetic field required for conversion of paramagnetic martensite into ferromagnetic austenite at the temperature of point $\mathrm{C}$ would be $\mu_{0} H_{c r} \sim 11 \mathrm{~T}$. Evidently, the given range of magnetic field change (from 0 to $\mu_{0} H=1.9 \mathrm{~T}$ ) is too weak to induce the transformation even at temperatures slightly below $M_{s}$. Therefore, the adiabatic temperature change measured in $\mathrm{Ni}_{50} \mathrm{Mn}_{36} \mathrm{Co}_{1} \mathrm{Sn}_{13}$ upon cooling (Fig. 5) originates from the magnetic subsystem alone due to the contributions from the inhomogeneous state composed of the austenite and martensite phases. Consequently, there is no sharp negative peak near $283 \mathrm{~K}$, in agreement with the experimental results of Fig. 5. As the temperature decreases between $M_{f}$ and $M_{s}$, a larger amount of ferromagnetic austenite is converted into the paramagnetic martensite phase. Then, the observed value of $\Delta T_{a d}^{\text {cooling }}$ changes gradually, when crossing the transition band between 290 and $280 \mathrm{~K}$, from the positive value of a pure ferromagnetic phase at $290 \mathrm{~K}$ to the small value of a pure paramagnet at $280 \mathrm{~K}$ (see Fig. 5).

In short, the apparent disagreement between $\Delta S_{m}$ and $\Delta T_{a d}\left(\Delta S_{m}^{\text {cooling }}>\Delta S_{m}^{\text {heating }}\right.$ whereas $\left|\Delta T_{a d}^{\text {cooling }}\right| \ll\left|\Delta T_{a d}^{\text {heating }}\right|$, see
Figs. 3 and 5) originates entirely from the strong hysteresis of the temperature- and magnetic-field-induced thermoelastic martensitic transformations in the $\mathrm{Ni}_{50} \mathrm{Mn}_{25+x} Z_{25-x}$ systems. On heating around $T_{M A}$, application of the magnetic field easily converts the paramagnetic martensite into ferromagnetic austenite, inducing a sharp and negative $\Delta T_{a d}^{\text {heating }}$ peak. On cooling around $T_{A M}$ at zero magnetic field, there is a temperature-induced conversion of the sample to the martensite phase. When the field is applied to measure $\Delta T_{a d}^{\text {cooling }}$, the converted sample does not return to the austenite phase and the slightly positive MCE is conditioned by the magnetic properties of both the phases.

\section{CONCLUSION}

In conclusion, our experimental results revealed that in the vicinity of the first-order martensitic phase transition of $\mathrm{Ni}_{50} \mathrm{Mn}_{36} \mathrm{Co}_{1} \mathrm{Sn}_{13}$, the magnitude of both isothermal magnetic entropy change, $\Delta S_{m}$, and adiabatic temperature change, $\Delta T_{a d}$, depend on the thermal history of the sample. Comparison of $\Delta S_{m}$ and $\Delta T_{a d}$ measured during heating and cooling processes, points to an apparent discrepancy between these characteristics of the MCE, i.e., $\Delta S_{m}^{\text {cooling }}>\Delta S_{m}^{\text {heating }}$ whereas $\left|\Delta T_{a d}^{\text {cooling }}\right| \ll\left|\Delta T_{a d}^{\text {heating }}\right|$. These features of the MCE in the studied alloy are essentially related to the hysteresis of the martensitic transition, which causes the magnetocaloric parameters to depend strongly on the temperature and field history of the experimental processes. In this paper it is fully proven that a thoughtful use of the phase diagram, as shown in Fig. 6, is necessary to understand the actual process during the measurement, and therefore, the results. These features are common for all the members of the family of $\mathrm{Ni}_{50} \mathrm{Mn}_{25+x} Z_{25-x}$ Heusler-based ferromagnetic shape memory alloys.

\section{ACKNOWLEDGMENTS}

This work was partially supported by RFBR (Grants No. 07-02-13629, No. 08-02-91317, and No. 09-02-01274), FANI (Grant No. 02.513.123097), the EU Seventh Framework Programme (Contract No. 214864), MICINN and FEDER funding of Projects No. MAT2007-61621, No. MAT2008-1077, and No. CSD2007-00010. Part of the work was carried out under the Collaborative Research Project of the Institute of Fluid Science, Tohoku University.
${ }^{1}$ Y. Sutou, Y. Imano, N. Koeda, T. Omori, R. Kainuma, K. Ishida, and K. Oikawa, Appl. Phys. Lett. 85, 4358 (2004).

${ }^{2}$ T. Krenke, M. Acet, E. F. Wassermann, X. Moya, L. Mañosa, and A. Planes, Phys. Rev. B 72, 014412 (2005).

${ }^{3}$ P. J. Brown, A. P. Gandy, K. Ishida, R. Kainuma, T. Kanomata, K.-U. Neumann, K. Oikawa, B. Ouladdiaf, and K. R. A. Ziebeck, J. Phys.: Condens. Matter 18, 2249 (2006).

${ }^{4}$ R. Kainuma, Y. Imano, W. Ito, H. Morito, Y. Sutou, K. Oikawa, A. Fujita, K. Ishida, S. Okamoto, O. Kitakami, and T. Kanomata, Appl. Phys. Lett. 88, 192513 (2006).
${ }^{5}$ T. Krenke, M. Acet, E. F. Wassermann, X. Moya, L. Mañosa, and A. Planes, Phys. Rev. B 73, 174413 (2006).

${ }^{6}$ R. Kainuma, Y. Imano, W. Ito, Y. Sutou, H. Morito, S. Okamoto, O. Kitakami, K. Oikawa, A. Fujita, T. Kanomata, and K. Ishida, Nature (London) 439, 957 (2006).

${ }^{7}$ K. Oikawa, W. Ito, Y. Imano, Y. Sutou, R. Kainuma, K. Ishida, S. Okamoto, O. Kitakami, and T. Kanomata, Appl. Phys. Lett. 88, 122507 (2006).

${ }^{8}$ W. Ito, Y. Imano, R. Kainuma, Y. Sutou, K. Oikawa, and K. Ishida, Metall. Mater. Trans. A 38, 759 (2007). 
${ }^{9}$ M. Khan, I. Dubenko, S. Stadler, and N. Ali, J. Phys.: Condens. Matter 20, 235204 (2008).

${ }^{10}$ L. Mañosa, X. Moya, A. Planes, O. Gutfleisch, J. Lyubina, M. Barrio, J. Tamarit, S. Aksoy, T. Krenke, and M. Acet, Appl. Phys. Lett. 92, 012515 (2008).

${ }^{11}$ J. Liu, N. Scheerbaum, J. Lyubina, and O. Gutfleisch, Appl. Phys. Lett. 93, 102512 (2008).

${ }^{12}$ T. Krenke, E. Duman, M. Acet, E. F. Wassermann, A. Moya, L. Mañosa, and A. Planes, Nature Mater. 4, 450 (2005).

${ }^{13}$ Z. D. Han, D. H. Wang, C. L. Zhang, S. L. Tang, B. X. Gu, and Y. W. Du, Appl. Phys. Lett. 89, 182507 (2006).

${ }^{14}$ M. Khan, N. Ali, and S. Stadler, J. Appl. Phys. 101, 053919 (2007).

${ }^{15}$ A. M. Tishin and Y. I. Spichkin, The Magnetocaloric Effect and its Applications (IOP, Bristol, 2003).

${ }^{16}$ T. Krenke, E. Duman, M. Acet, X. Moya, L. Mañosa, and A. Planes, J. Appl. Phys. 102, 033903 (2007).

${ }^{17}$ B. Gao, F. X. Hu, J. Shen, J. Wang, J. R. Sun, and B. G. Shen, J. Magn. Magn. Mater. 321, 2571 (2009).

${ }^{18}$ B. Hernando, J. L. Sánchez Llamazares, J. D. Santos, V. M. Prida, D. Baldomir, D. Serantes, R. Varga, and J. González,
Appl. Phys. Lett. 92, 132507 (2008).

${ }^{19}$ X. Moya, Ll. Mañosa, A. Planes, T. Krenke, E. Duman, M. Acet, and E. F. Wassermann, J. Magn. Magn. Mater. 316, e572 (2007).

${ }^{20}$ A. Planes, L. Mañosa, and M. Acet, J. Phys.: Condens. Matter 21, 233201 (2009).

${ }^{21}$ Z. D. Han, D. H. Wang, C. L. Zhang, H. C. Xuan, J. R. Zhang, B. X. Gu, and Y. W. Du, J. Appl. Phys. 104, 053906 (2008).

${ }^{22}$ S. Yu. Dan'kov, A. M. Tishin, V. K. Pecharsky, and K. A. Gschneidner, Jr., Rev. Sci. Instrum. 68, 2432 (1997).

${ }^{23}$ R. Y. Umetsu, R. Kainuma, Y. Amako, Y. Taniguchi, T. Kanomata, K. Fukushima, A. Fujita, K. Oikawa, and K. Ishida, Appl. Phys. Lett. 93, 042509 (2008).

${ }^{24}$ V. V. Khovaylo, T. Kanomata, T. Tanaka, M. Nakashima, Y. Amako, R. Kainuma, R. Y. Umetsu, H. Morito, and H. Miki, Phys. Rev. B 80, 144409 (2009).

${ }^{25}$ M. Pasquale, C. P. Sasso, L. Giudici, T. Lograsso, and D. Schlagel, Appl. Phys. Lett. 91, 131904 (2007).

${ }^{26}$ V. K. Pecharsky and K. A. Gschneidner, Jr., J. Appl. Phys. 90, 4614 (2001). 